\title{
"O que vêm da terra não faz mal" - relatos de problemas relacionados ao uso de plantas medicinais por raizeiros de Diadema/SP
}

\author{
Juliana Lanini*, Joaquim M. Duarte-Almeida, Solange Nappo, Elisaldo A. Carlini
}

Departamento de Psicobiologia, Universidade Federal de São Paulo, Rua Botucatu 862, 04023-062 São Paulo-SP, Brasil

\begin{abstract}
RESUMO: A crença de que medicamentos à base de plantas são isentos de riscos à saúde faz parte da bagagem cultural da população afeita ao seu uso: "o que vêm da terra não faz mal”. No entanto, o potencial tóxico, as características específicas do usuário, a possibilidade de contaminação e a falta de regulamentação constituem fatores de risco para a ocorrência de reações adversas, intoxicações e outras complicações decorrentes de seu uso. Foram realizadas entrevistas semi-estruturadas junto a 20 raizeiros (vendedores de ervas "in natura") na cidade de Diadema/SP. Foram relatados 40 casos de problemas relacionados ao uso de 22 espécies de plantas medicinais. As espécies mais citadas foram Luffa operculata - buchinha (7 casos), Senna alexandrina - sene (4 casos) e Paullinia cupana - guaraná (3 casos). Dentre os sinais e sintomas relatados, os mais freqüentes foram relacionados ao sistema nervoso central, problemas gastrointestinais e cardiovasculares. Os entrevistados também relataram um caso de aborto relacionado ao uso de $S$. alexandrina e três casos de óbito após a ingestão do chá do fruto de $L$. operculata. O caráter "natural" das plantas medicinais não é sinônimo de ausência de riscos para a população usuária. É necessária a implantação de políticas de fitofarmacovigilância eficientes, a fim de tornar o consumo mais racional e, deste modo, minimizar os riscos à população usuária.
\end{abstract}

Unitermos: Reações adversas, intoxicações, plantas medicinais, fitofarmacovigilância, entrevistas semi-estruturadas.

\begin{abstract}
Natural and therefore free of risks" - adverse effects, poisonings and other problems related to medicinal herbs by "raizeiros" in Diadema/SP". Traditionally, medicinal herbs have been considered gentle and harmless because of their natural origin: "natural and therefore free of risks". However, toxic potential, various consumer-related factors (such as age, disease factors, pregnancy, etc), contamination, and lack of regulation concerning quality control are some of the risk factors deemed to be associated with herbal adverse reactions, poisonings and other complications. Over a 7-month period, semi-structured interviews were carried out with 20 "raizeiros" (traditional healers) in Diadema/SP. We found 40 unexpected events related to 22 species of medicinal herbs. The most frequent species reported were Luffa operculata "buchinha" (7 reports), Senna alexandrina - "sene" (4 reports) and Paullinia cupana - "guaraná" (3 reports). Among the adverse events, the most frequent signs and symptoms reported involved the central nervous, gastrointestinal and cardiovascular systems. They also reported one case of abortion (related with S. alexandrina) and three deaths related with L. operculata. Adverse events that result from "natural" medicines are relatively uncommon, but they certainly occur more often than acknowledged. Medicinal plants are traditionally considered as innocuous, but as commercially available medicinal products they require sanitary surveillance in order to identify their risks, to give them a legal status, to evaluate their efficacy and to ascertain their safety.
\end{abstract}

Keywords: Adverse reactions, poisonings, medicinal herbs, phytopharmacovigilance, semistructured interviews.

\section{INTRODUÇÃO}

A crença de que medicamentos à base de plantas são isentos de riscos à saúde faz parte da bagagem cultural da população afeita ao seu uso. No entanto, o caráter "natural" de tais produtos não é garantia da isenção de reações adversas e outros problemas decorrentes de tal medicina. A falta de regulamentação e controle na comercialização, o fácil acesso, o risco de contaminação e/ou adulteração do material (Ko, 1998) e as características específicas dos usuários (De Smet, 2004) constituem fatores de risco à ocorrência de reações adversas e outros problemas oriundos ao seu uso.

A credulidade de que "o que vem da terra não faz mal" vem sendo desmentida cientificamente há vários anos em muitos países (Arseculeratne et al, 1985; MacGregor et al., 1989; Shah et al., 1989; 
Godlee, 1992; De Smet, 1997; Stewart et al., 1998; Stickel et al., 2005; Amorim et al., 2007; Alexandre et al., 2008a,b; Marliére et al., 2008; Silveira et al., 2008; Veiga-Junior et al., 2008). Encontram-se na literatura científica relatos de complicações cardíacas, hepáticas (Woolf et al., 1994; Lai \& Chan, 1999), renais (Abt et al., 1995), hematológicas (Ries \& Sahud, 1975; Chan et al., 1977; Ko, 1998) e intestinais (Sossai et al., 2007) por fitoterápicos.

Também vale ressaltar que tanto a planta medicinal quanto os produtos de sua biotransformação são agentes xenobióticos e, portanto, potencialmente tóxicos, não tendo somente efeitos imediatos $\mathrm{e}$ facilmente correlacionáveis com a sua ingestão; mas também efeitos que se instalam a longo prazo e de forma assintomática (De Smet, 2004).

Em 2006 foi aprovada a Política Nacional de Plantas Medicinais e Fitoterápicos (Brasil, 2006) onde ficou estipulada a inserção de terapias alternativas e práticas populares (entre elas a fitoterapia) no Sistema Único de Saúde (SUS). Desde então, práticas relacionadas à distribuição de fitoterápicos e a implantação das "Farmácias Vivas" (Matos, 1998) vêm se tornando uma realidade no país. Sendo assim, a necessidade de estudos toxicológicos e a implantação de políticas de fitofarmacovigilância devem ser tomadas como prioridade para a saúde pública.

Nesta perspectiva, o presente estudo teve como principal objetivo identificar e descrever, através de técnicas qualitativas, as possíveis reações adversas, bem como casos de intoxicações e outros problemas decorrentes do uso de plantas medicinais junto aos raizeiros (vendedores de erva in natura) da cidade de Diadema/SP.

\section{MATERIAL E MÉTODOS}

Tratando-se de fenômeno pouco conhecido e dependente da cultura local (Diadema) foram utilizados no presente estudo os princípios da metodologia qualitativa, ou seja, as informações estão com aqueles que são associados ao fenômeno. No caso, foram contatados os raizeiros e ainda, o critério numérico não foi privilegiado mas os significados identificados nos relatos desses raizeiros (Mays \& Pope, 1995; Pope et al., 2000, Patton, 2001). Dessa forma os resultados alcançados levaram em consideração os valores, crenças, impressões e opiniões dessa população (Minayo \& Sanchez, 1993; Nogueira-Martins \& Bógus, 2004). Técnicas da investigação qualitativa foram utilizadas na construção da amostra e no levantamento das informações a partir dos constituintes da mesma:

\section{Amostra}

Amostra intencional, selecionada por critérios (Amostra por critérios), constituída de 20 raizeiros
(Patton, 2001), recrutados através da técnica de snowball (Biernacki \& Waldorf, 1981) e que cumpriram o critério estabelecido, ou seja, com tempo de pelo menos 2 anos de experiência na venda de plantas medicinais e/ou tradição de família no ramo. Esse critério garantiu que fizessem parte da amostra os casos ricos em informação (Victora et al., 2000). A seleção dos raizeiros respeitou os princípios da aleatoriedade e voluntariado, sendo direito de todos os entrevistados a retirada de sua participação durante a coleta de dados, a qual foi iniciada após parecer favorável do Comitê de Ética em Pesquisa da UNIFESP (CEP 1803/07).

\section{Tamanho da amostra}

Um $\mathrm{N}=20$ raizeiros provem da totalidade de raizeiros de Diadema que cumpre o critério para inclusão na amostra (WHO, 1994).

\section{Entrevistas}

Semi-estruturadas, anônimas e gravadas com a prévia concordância dos entrevistados.

\section{Guia de questões}

O entrevistador utilizou-se de um guia com temas para perguntas (Patton, 2001). As mesmas exploraram o conhecimento dos entrevistados sobre a possível relação entre plantas medicinais e eventos adversos, $\mathrm{O}$ foco de atenção foi o relato de casos de reações adversas, intoxicações ou quaisquer problemas possivelmente relacionados ao uso de plantas medicinais de conhecimento dos raizeiros.

\section{Análise dos resultados}

Nessa fase, os passos recomendados por Minayo (1994) e Bardin (2004), foram utilizados. As entrevistas gravadas foram transcritas e uma leitura flutuante inicial foi feita de forma o entrevistador entrar em contato com o material, sendo este desmembrado de acordo com os temas investigados. Para cada tema foram associadas as respostas respectivas dos 20 entrevistados. Nessa fase o entrevistador identificou as unidades dos textos de relevância para o tema. Os resultados são apresentados de acordo com sua freqüência em números absolutos e/ou porcentagens.

Uma vez que os dados obtidos no presente trabalho são provenientes dos relatos dos entrevistados, o estabelecimento das relações de causalidade (Karch \& Lasagna, 1975) torna-se mais complexo. Mesmo assim, classificaram-se os eventos da seguinte maneira: a) Reação Adversa: casos em que foi relatada a ocorrência de sinais e sintomas indesejáveis após a administração da dose recomendada, uso e forma de preparo corretos (de acordo com as instruções dadas 
pelo prescritor);

b) Intoxicação: casos em que foi relatado o uso de doses e /ou quantidades elevadas (além do recomendado) pelos consumidores; ou casos em que há suspeita de contaminação do material;

c) Outros: casos em que o material vegetal foi preparado e/ou consumido de forma inadequada (excluindo-se os casos de superdosagem).

Uma pesquisa utilizando as bases de dados PubMed e Scopus foi realizada em busca de trabalhos de toxicologia pré-clínica e relatos de casos que viessem de encontro às informações obtidas junto aos raizeiros. A nomenclatura científica das plantas citadas foi sugerida a partir de sua denominação popular e indicação de uso de acordo com Pio Correia (1984) e bancos de dados acima mencionados. Uma vez que uma mesma denominação popular pode ser atribuída a diferentes espécies vegetais, adotou-se como critério de escolha para nomenclatura aqui descrita aquela encontrada na maioria dos trabalhos científicos consultados.

\section{RESULTADOS}

Treze dos entrevistados (65\%) eram do sexo masculino. Com relação à procedência dos entrevistados, $12(60 \%)$ emigraram da região Nordeste. Seis entrevistados eram da região Sudeste e dois da região Centro Oeste. Quatorze $(70 \%)$ entrevistados declararam ter tradição familiar no comércio de ervas medicinais, sendo esta a origem de seu conhecimento no assunto. Os demais $(30 \%)$ citaram livros, revistas e a mídia como principais fontes de conhecimento. Dezessete entrevistados (85\%) tinham acima de 40 anos. Quanto ao tempo de trabalho, 14 entrevistados (70\%) apresentavam mais de 10 anos de experiência e o restante possuía entre dois e nove anos de experiência.

Treze dos entrevistados relataram ter conhecimento sobre casos relacionados ao tema estudado, totalizando 40 casos de problemas relacionados ao uso de 22 espécies de plantas medicinais. Em 36 casos (90\%), a forma de preparo do material vegetal foi o chá, e a parte da planta mais utilizada foi a folha, com 23 citações $(57,5 \%)$.

A Tabela 1 mostra a classificação dos eventos relacionados a cada planta, o número de vezes que uma dada espécie foi citada, os sinais e sintomas descritos e os trabalhos existentes na literatura que corroboram a possível relação entre os eventos citados e a planta em questão. Os termos empregados na tabela não reproduzem fidedignamente o discurso dos entrevistados, tendo sido substituídos por termos técnicos genéricos correspondentes. Por exemplo, para "colou os intestinos" usou-se distúrbios gastrintestinais;

"urina solta" foi descrito como incontinência urinária; "acelerou o coração", como taquicardia, etc.

As plantas com maior número de citações relacionadas à reações adversas e/ou intoxicações foram Luffa operculata (L.) Cogn., Senna alexandrina. Mill. e Paullinia cupana Kunth, com sete, quatro e três citações, respectivamente. Com relação ao tipo de problema ocorrido, foram classificados um total de 20 casos de reações adversas (50\%), 15 casos de intoxicações $(37,5 \%)$ e cinco casos $(12,5 \%)$ onde o problema foi relacionado ao uso incorreto (excluindo-se os casos de superdosagem) do material vegetal.

Quatro citações foram relacionadas ao uso de folhas frescas ao invés de folhas secas, onde dois casos envolveram chá das folhas de amora (Morus nigra L.), um foi relacionado ao chá das folhas de erva-doce (Pimpinella anisum L.), e um quarto caso para chá das folhas de abacate (Persea americana Mill.). Em um dos casos foi descrito o consumo inadequado do chá de pautenente (Quassia amara L.), que teria sido consumido muito quente e após uma grande refeição.

\section{DISCUSSÃO}

Como o presente trabalho versa sobre o tema sob a ótica apenas dos raizeiros, dados mais detalhados sobre os pacientes (seu histórico de doenças, uso concomitante de outras substâncias, hábitos de vida, relação de tempo entre a ingestão do medicamento e o aparecimento dos problemas) não puderam ser desvendados. Toda a classificação dos eventos aqui apresentados se baseou exclusivamente naquilo que os entrevistados declararam durante a pesquisa. Uma vez que não há dados definidos cientificamente sobre posologia e forma de uso dos medicamentos aqui estudados, adotou-se como "uso correto" aquele uso indicado pelo próprio raizeiro no momento da venda do material vegetal. Aqui vale ressaltar o papel destes indivíduos como um "elo" entre a população usuária e as plantas medicinais. De acordo com a definição encontrada no Dicionário Aurélio, "raizeiro" é o substantivo masculino que designa "curandeiro que trata doenças valendo-se de raízes vegetais", sendo também chamados pela população de "ervatários", que de acordo com o mesmo dicionário são definidos como "indivíduos que nos campos e matas colhem ervas medicinais para vender nos herbanários ou a retalho". No caso do presente estudo, denominamos "raizeiro" o indivíduo que comercializa ervas medicinais "in natura" e que atende aos critérios de inclusão na amostra estabelecidos para a pesquisa. De fato, os raizeiros detêm o conhecimento sobre o "poder de cura" associado às plantas, sua forma de uso, posologia, etc; baseado na sua própria experiência e em informações adquiridas empiricamente; sendo reconhecidos pela população usuária como uma espécie de "médico", capaz de indicar a erva correta para o tratamento de determinados males. Sendo assim, utilizou-se esse conhecimento como instrumento de classificação para os eventos relatados.

Também não foi possível a identificação 
adequada das plantas citadas, dado que os casos relatados aconteceram no passado, muitas vezes em outras regiões do país, sendo impossível a coleta, identificação e análise do material que de fato esteve envolvido no evento.

A planta mais citada, L. operculata, está entre as dez plantas mais utilizada nas tentativas de aborto no Brasil (Mengue et al., 1997). Em levantamento realizado no Hospital das Clínicas de Ribeirão Preto, a buchinha foi responsável por $4,67 \%$ dos casos de intoxicações por plantas atendidas no período de janeiro de 1995 e setembro de 2000 (Oliveira et al., 2003). Segundo os raizeiros, três dos sete casos citados evoluíram a óbito (por hemorragia vaginal após ingestão do chá dos frutos para provocar aborto) e um caso a má formação fetal. Neste caso foi relatada a ingestão do chá do fruto num estágio mais avançado da gestação (acima de três meses), quando este não teria mais a capacidade de expulsar o embrião. Além de seu uso como abortiva, a buchinha também é utilizada em inalações no tratamento da sinusite e congestão nasal. Três relatos de hemorragia nasal e cefaléia foram mencionados pelos raizeiros. A ação tóxica de $L$. operculata pode estar relacionada ao efeito de cucurbitacinas (Miró, 1995), mas ainda não há total certeza sobre o assunto. Em estudo recente, a administração do decocto de L.operculata reduziu significativamente a taxa de natalidade em camundongos fêmeas no período da implantação dos embriões (Barilli et al., 2005). Menon-Miyake et al. (2005) encontraram alterações estruturais e microscópicas importantes no epitélio respiratório de sapos quando estes eram expostos a diferentes concentrações de L. operculata.

Quatro citações foram relacionadas ao uso do sene, S. alexandrina. Os entrevistados apontaram o sene como sendo uma "planta perigosa", que deve ser ingerida em pequenas quantidades. Dois casos foram descritos como intoxicações, caracterizados pela ingestão de grande quantidade de chá por longos períodos de tempo, causando, segundo os entrevistados, "colamento do intestino" e câncer de colón. Mereto et al. (1996) e Mascolo et al. (1999) encontraram relação entre a administração de altas doses de $S$. alexandrina e a ocorrência de câncer de colón em ratos. De fato, é possível que antraquinonas presentes no sene possam ser um fator de risco para o desenvolvimento de câncer de colón (Siegers et al., 1993). No entanto, deve-se considerar fatores relacionados ao histórico dos pacientes em questão, seus hábitos alimentares, uso concomitante de outras substancias laxativas, severidade do quadro de constipação e histórico familiar.

O sene também foi relacionado a um caso de aborto. Problemas de constipação são muito comuns durante a gravidez (Keller et al., 2008). Segundo Shulz et al. (2002), produtos que possuem antraquinonas em sua composição devem ser evitados durante a gravidez, uma vez que existe o risco de perda do feto devido ao aumento do fluxo sanguíneo para o útero e indução de contrações.

Com relação aos problemas relacionados ao uso de $P$. cupana (guaraná), em um dos casos foi relatado que o usuário consumiu grande quantidade do pó de guaraná, tendo como conseqüência um quadro de alucinações, tontura, vômito e taquicardia. Nos outros dois casos, sintomas como enjôo, taquicardia e cefaléia foram relacionados ao uso do guaraná em pó, mesmo quando administrado na dose considerada "correta" pelos raizeiros. Em ambas as situações, os sinais e sintomas apresentados podem estar relacionados à cafeína.

A cafeína é a droga psicoativa mais utilizada no mundo e está presente em diversas fontes de alimentos, além de ser um dos principais compostos presentes nas fórmulas emagrecedoras (Gilbert, 1984). Análises fitoquímicas mostraram que as sementes do guaraná podem conter de 4 a $8 \%$ de cafeína (Proença da Cunha et al. 2005); o que associado à ingestão de outras fontes dessa substância, pode levar a um quadro de intoxicação. Relatos de casos com sintomas semelhantes aos descritos pelos entrevistados já foram descritos na literatura (Cannon et al., 2001; Baghkhani \& Jafari, 2002; Schmidt \& Karlson-Stiber, 2008). Quadros de necrose do miocárdio, hemorragia aguda e morte súbita foram encontrados em ratos que receberam guaraná e efedra (Ephedra sinica Stapf) concomitantemente (Nyska et al., 2005).

Com relação aos casos de uso das folhas frescas ao invés de secas, os entrevistados afirmaram que as folhas frescas apresentam maior concentração de princípios ativos e por isso são potencialmente tóxicas, tendo sido este o motivo do aparecimento dos sintomas desagradáveis. De fato, durante o processo de secagem do material vegetal pode haver perda de princípios ativos voláteis e/ou termolábeis (Borges et al., 2005). Com exceção de $P$. anisum (erva-cidreira), não existem evidências científicas a respeito da possibilidade de tais perdas nas outras espécies citadas.

Em um dos casos de intoxicação, o entrevistado relatou ter vendido, sem saber, um pacote de chá emagrecedor ("Mistura emagrecedora", contendo Anona muricato L., Chiococca brachiata Ruiz \& Pav., Casearia sylvestris Sw., Baccharis trimera L. e Senna alexandrina. Mill. ) contaminado por fungos. Segundo ele, o material não estava suficientemente seco e, após ter sido colocado em saco plástico fechado, "fungou". Uma vez que não há um controle efetivo sobre cultivo, colheita, secagem e envasamento das plantas comercializadas nos casos aqui descritos, o risco de contaminação é alto, já tendo sido descrito por diversos autores (Ko, 1998; Melo et al., 2007).

Entre 1968 e 1997, a OMS recebeu um total de 8985 relatos de eventos adversos relacionados ao uso de plantas medicinais (Farah \& Edwards, 2000). Embora esse número seja apenas uma pequena fração do mesmo dado relativo a medicamentos alopáticos, deve-se levar 


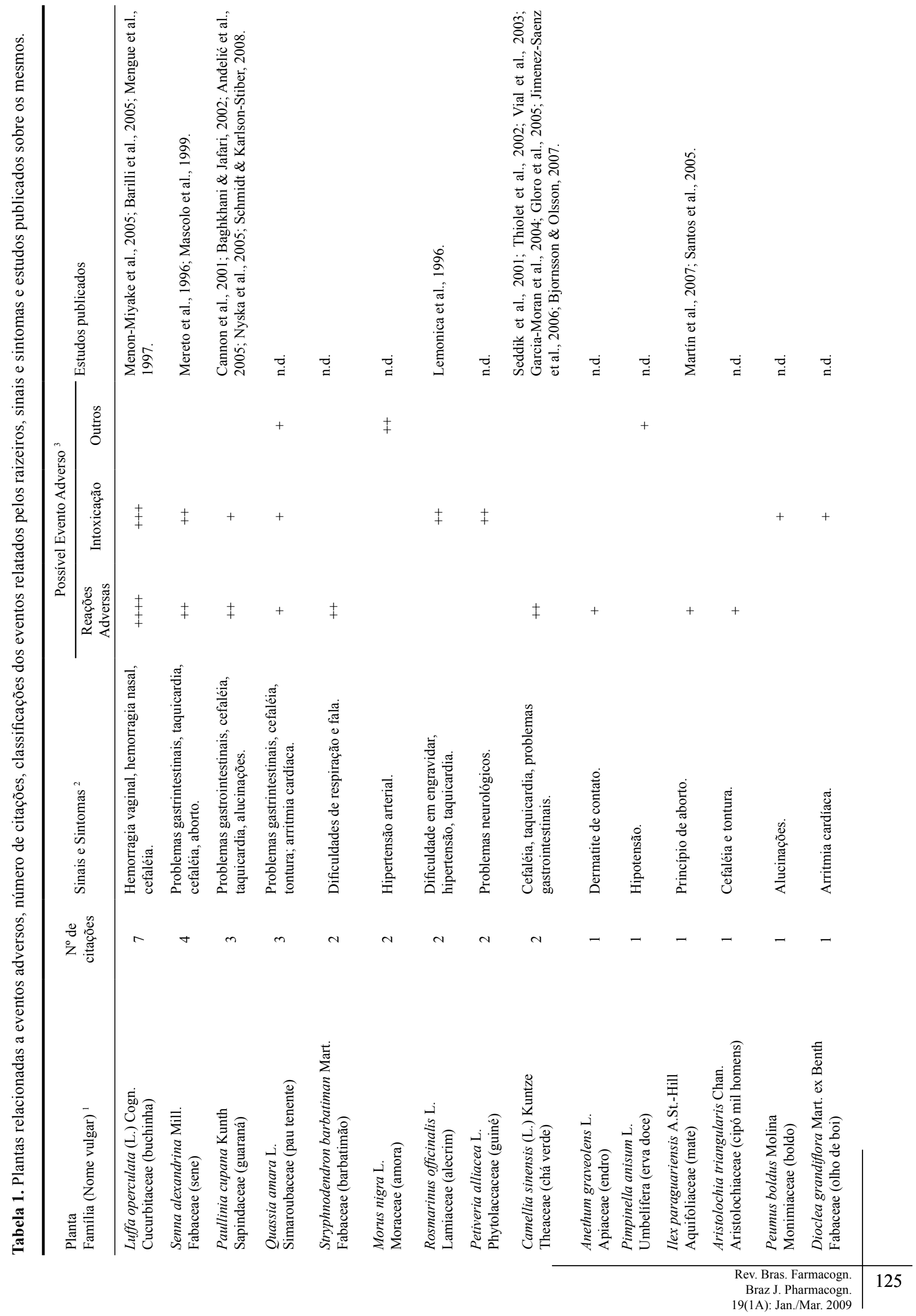




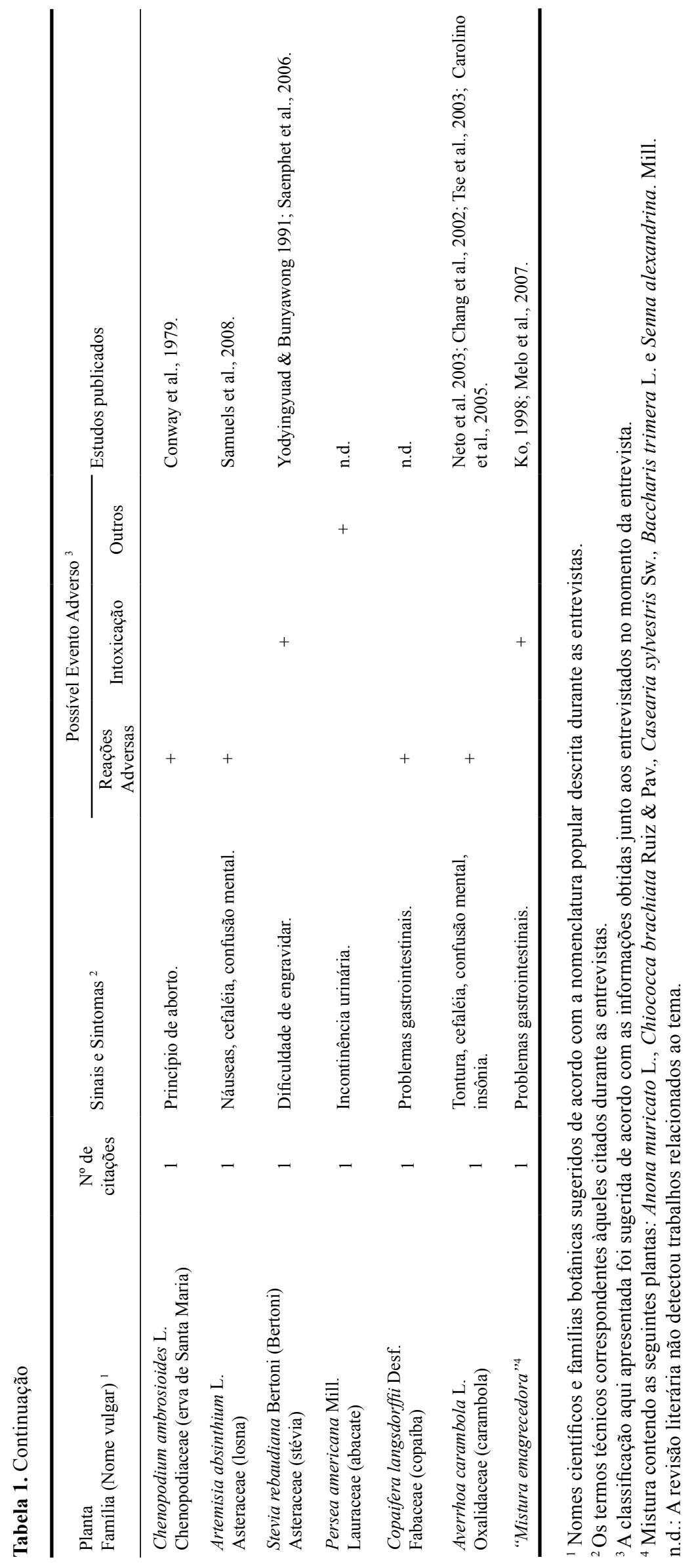


em conta a inexistência de uma cultura de denúncia com relação a tais produtos e também a ausência de órgãos capazes de centralizar e monitorar tais informações.

$\mathrm{O}$ mercado consumidor de tais produtos cresce a cada ano (Ernst, 2002; Carvalho et al., 2008), milhares de sites na internet anunciam a "cura natural através das plantas" como um caminho eficaz e seguro aos pacientes, que não raramente substituem terapias convencionais de eficácia comprovada por fármacos ainda não estudados (Wong \& Castro, 2003).

Diante do aqui exposto, fica claro que os riscos relacionados ao uso de plantas medicinais são uma realidade da qual não se pode mais fugir. Sendo assim, são necessários maiores estudos a fim de explorar os potenciais terapêuticos e verificar a toxicidade e possíveis riscos relacionados às plantas medicinais utilizadas pela população. Nota-se também a necessidade da implantação de políticas de fitofarmacovigilância eficientes, a fim de tornar seu consumo mais racional e, deste modo, minimizar os riscos à população usuária.

\section{AGRADECIMENTOS}

Aos entrevistados, à Lígia Schaefer de Almeida (aluna de iniciação científica/PIBIC), aos pesquisadores do CEBRID (Ricardo Tabach, Fúlvio Mendes, Yone Moura e Zila Sanchez), à FAPESP, PRODOC/CAPES e ao $\mathrm{CNPq}$ pelo apoio financeiro.

Este trabalho foi parcialmente apoiado pela FAPESP (processo 07/51887-4) e com bolsa de pósgraduação, nível mestrado fornecida pelo $\mathrm{CNPq}$

\section{REFERÊNCIAS}

Abt A, OH JY, Huntington RA, Burkhart KK 1995. Chinese herbal medicine induced acute renal failure. Arch Inter Med 155: 211-212.

Alexandre RF, Bagatini F, Simões CMO 2008a. Interações entre fármacos e medicamentos fitoterápicos à base de ginkgo ou ginseng. Rev Bras Farmacogn 18: 117126.

Alexandre RF, Bagatini F, Simões CMO 2008b. Potenciais interações entre fármacos e produtos à base de valeriana ou alho. Rev Bras Farmacogn 18: 455-463.

Amorim MFD, Diniz MFFM, Araújo MST, Pita JCLR, Dantas JG, Ramalho JA, Xavier AL, Palomaro TV, Júnior NLB 2007. The controvertible role of kava (Piper methysticum G. Foster) an anxiolytic herb, on toxic hepatitis. Rev Bras Farmacogn 17: 448-454.

Anđelić S, Tamburkovski V, Cimbaljević N, Žegarac D 2005. Cardiac arrest in asthmatic patient after consummation Guarana. Timoc Med Glas 30: 78-81.

Arseculeratne SN, Gunatilaka AAL, Panabokke RG 1985. Studies on medicinal plants of Sri Lanka Part 14: Toxicity of some traditional medicinal herbs. $J$ Ethnopharmacol 13: 323-335.

Baghkhani L, Jafari M 2002. Cardiovascular adverse reactions associated with guarana: Is this a causal effect? $J$ Herb Pharmacother 2: 57-61.
Bardin L 2004. Análise de Conteúdo. $3^{\mathrm{a}}$ ed. Lisboa: Edições 70.

Barilli SLS, Santos ST, Montanari T 2005. Efeito do decocto dos frutos de buchinha-do-norte (Luffa operculata Cogn.) sobre a reprodução feminina e o desenvolvimento embrionário e fetal. XVII Salão de Iniciação Científica da UFRGS. Porto Alegre, Brasil.

Biernacki P, Waldorf D 1981. Snowball sampling: problems and techniques of chain referral sampling. Sociol Meth Res 10: 141-163.

Bjornsson E, Olsson R 2007. Serious adverse liver reactions associated with herbal weight-loss supplements. $J$ Hepatol 47: 295-297.

Borges DB, Farias MR, Simões CMO, Schenkel EP 2005. Comparação das metodologias da Farmacopéia Brasileira para determinação de água em matériasprimas vegetais, e validação da determinação de água em analisador de umidade para Calendula officinalis L., Foeniculum vulgare Miller, Maytenus ilicifolia Mart. ex Reissek e Passiflora alata Curtis. Rev Bras Farmacogn 15: 229-236.

Brasil 2006. Presidência da República. Decreto $n^{\circ} 5813$ de 22 de junho de 2006. Aprova a Política Nacional de Plantas Medicinais e Fitoterápicos e dá outras providências. DOU. Poder Executivo, Brasília, DF, 23 de junho 2006.

Carolino RO, Beleboni RO, Pizzo AB, Vecchio FD, GarciaCairasco N, Moyses-Neto M, Santos WF, CoutinhoNetto J 2005. Convulsant activity and neurochemical alterations induced by a fraction obtained from fruit Averrhoa carambola (Oxalidaceae: Geraniales). Neurochem Int 46: 523-531.

Cannon ME, Cooke CT, McCarthy JS 2001. Caffeine-induced cardiac arrhythmia: An unrecognized danger of health food products. Med J Aust 174: 520-521.

Carvalho ACB, Balbino EE, Maciel A, Perfeito JPS 2008. Situação do registro de medicamentos fitoterápicos no Brasil. Rev Bras Farmacogn 18: 314-319.

Chan H, Yeh YY, Billmeier GJ, Evens WE 1977. Lead poisoning from ingestion of Chinese herbal medicine. Clin Toxicol 10: 273-281.

Chang CT, Chen YC, Fang JT, Huang CC 2002. Star fruit (Averrhoa carambola) intoxication: an important cause of consciousness disturbance in patients with renal failure. Ren Fail 24: 379-382.

Conway GA, Slocumb JC 1979. Plants used as abortifacients and emmenagogues by Spanish New Mexicans. $J$ Ethnopharmacol 1: 241-261.

De Smet PAGM 1997. Efectos adversos de los remedios herbarios. Adverse Drug React Bull 16: 38-43.

De Smet PAGM 2004. Health risks of herbal remedies: An update. Clin Pharmacol Ther 76: 1-17.

Ernst E 2002. The risk-benefit profile of commonly used herbal therapies: Ginkgo, St. John's wort, Ginseng, Echinacea, Saw Palmetto, and Kava. Ann Intern Med 36: 42-53.

Farah MH, Edwards R 2000. International monitoring of adverse health effects associated with herbal medicines. Pharmacoepidemiol Drug Safety 9: 10512.

Garcia-Moran S, Saez-Royuela F, Gento E, Lopez MA, Arias L 2004. Acute hepatitis associated with Camellia thea and Orthosiphon stamineus ingestion. Gastroenterol 
Hepatol 27: 559-560.

Gilbert RM 1984. Caffeine consumption. In: Spiller GA (org) The methylxanthine beverages and foods: chemistry, consumption, and health effects. New York: Liss, 185-213.

Gloro R, Hourmand-Ollivier I, Mosquet B, Mosquet L, Rousselot P, Salame E, Piquet MA, Dao T 2005. Fulminant hepatitis during self-medication with hydroalcoholic extracts of green tea. Eur $J$ Gastroenterol Hepatol 17: 1135-1137.

Godlee F 1992. Medicinal plants: another man's poison. BMJ 305: 1583-1585.

Jimenez-Saenz M, del Carmen M 2006. Acute hepatitis associated with the use of green tea infursion. $J$ Hepatol 44: 616-619.

Karch FE, Lasagna L 1975. Adverse drug Reactions. A critical review. JAMA 234: 1236-1241.

Keller J, Frederking D, Layer P; Medscape 2008. The spectrum and treatment of gastrointestinal disorders during pregnancy. Nat Clin Pract Gastroenterol Hepatol 5: 430-443.

Ko RJ 1998. Adulterants in Asian patent medicines. $N$ Engl Med 339: 847.

Lai CK, Chan AYW 1999. Tetrahydropalmatine poisoning: diagnosis of nine adult overdoses based on toxicology screens by HPLC with diode-array detection and gas chromatography-mass spectrometry. Clin Chem 45 : 229-236.

Lemonica IP, Damasceno DC, Di Stasi LC 1996. Study of the embryotoxic effects of an extract of rosemary (Rosmarinus officinalis L.). Braz J Med Biol Res 29: 223-227.

MacGregor FB, Abernethy VE, Dahabra S, Cobden I, Hayes PC 1989. Hepatotoxicity of some herbal remedies. BJM 299: 1156-1157.

Marliére LDP, Ribeiro AQ, Brandão MGL, Klein CH, Acurcio FA 2008. Utilização de fi toterápicos por idosos: resultados de um inquérito domiciliar em Belo Horizonte (MG), Brasil. Rev Bras Farmacogn 18 (Supl.): 754-760.

Martín I, López-Vílchez MA, Mur A, García-Algar O, Rossi S, Marchei E, Pichini S 2007. Neonatal withdrawal syndrome after chronic maternal drinking of mate. Ther Drug Monit 29: 127-129.

Mascolo N, Mereto E, Borrelli F, Orsi P, Sini D, Izzo AA, Massa B, Boggio M, Capasso F 1999. Does Senna extract promote growth of aberrant crypt foci and malignant tumors in rat colon? Digest Dis Sci 44: 2226-2230.

Matos FJA 1998. Farmácias Vivas: Sistema de Utilização de Plantas Medicinais projetado para pequenas comunidades. 3.ed. Fortaleza: Edições USCE.

Mays N, Pope C 1995. Rigour and qualitative research. Brit Med J 311: 109-112.

Melo JG, Martins JDGR, Amorim ELC, Albuquerque UP 2007. Qualidade de produtos a base de plantas medicinais comercializados no Brasil: castanhada-índia (Aesculus hippocastanum L.), capimlimão (Cymbopogon citratus (DC.) Stapf e centela (Centella asiatica (L.) Urban). Acta Bot Bras 21: 27-36.

Mengue SS, Schenkel EP, Mentz LA, Schmidt MI 1997. Especies vegetales utilizadas por embarazadas con el objeto de provocar la menstruación (Encuesta a siete ciudades de Brasil). Acta Farm Bonaerense 16: 251-258.

Menon-Miyake MA, Saldiva PHN, Lorenzi-Filho G, Ferreira MA, Butugan O, Oliveira RCO 2005. Luffa operculata effects on the epithelium of frog palate: histological features. Rev Bras Otorrinolaringol 71: 132-138.

Mereto E, Ghia M, Brambilla G 1996. Evaluation of the potential carcinogenic activity of Senna and Cascara glycosides for the rat colon. Cancer Lett 101: 79-83.

Minayo MCS, Sanches O 1993. Quantitativo-qualitativo: Oposição ou complementariedade? Cad Saúde Pública 9: 239-262.

Minayo MCS 1994. O desafio do conhecimento: pesquisa qualitativa em saúde. São Paulo-Rio de Janeiro: Hucitec-Abrasco.

Miró M 1995. Cucurbitacins and their pharmacological effects. Phytother Res 9: 159-168.

Neto MM, da Costa JA, Garcia-Cairasco N, Netto JC, Nakagawa B, Dantas M 2003. Intoxication by star fruit (Averrhoa carambola) in 32 uraemic patients: treatment and outcome. Nephrol Dial Transpl 18: 120-125.

Nogueira-Martins MCF, Bógus CM 2004. Considerações sobre a metodologia qualitativa como recurso para o estudo das ações de humanização em saúde. Saúde e Sociedade. Rev Colomb Obstet Ginecol 13: 44-57.

Nyska A, Murphy E, Foley JF, Collins BJ, Petranka J, Howden R, Hanlon P, Dunnick JK 2005. Acute hemorrhagic myocardial necrosis and sudden death of rat exposed to a combination of ephedra and caffeine. Toxicol Sci 83: 388-396.

Oliveira RB, Godoy SAP, Costa FB 2003. Plantas tóxicas conhecimento e prevenção de acidentes. v.1. Ribeirão Preto: Holos Editora.

Patton MQ 2001. Qualitative Research \& Evaluation Methods. 3.ed. London: Sage Publications.

Pio Corrêia M 1984. Dicionário das plantas úteis do Brasil e das exóticas cultivadas. Rio de Janeiro: IBDF.

Pope C, Ziebland S, Mays N 2000. Analysing qualitative data. Brit Med J 320: 114-116.

Proença da Cunha A, Salgueiro L, Roque OR 2005. Metilxantinas In: Proença da Cunha (Ed) Farmacognosia e fitoquímica, Lisboa: Fundação Calouste Gulbenkian, 595-605.

Ries CA, Sahud MA 1975. Agranulocytosis caused by Chinese herbal medicines. JAMA 231: 352-355.

Saenphet K, Aritajat S, Saenphet S, Manosroi J, Manosroi A 2006. Safety evaluation of aqueous extracts from Aegle marmelos and Stevia rebaudiana on reproduction of female rats. Southeast Asian J Trop Med Public Health 37: 203-205.

Samuels N, Finkelstein Y, Singer, SR, Oberbaum, M. 2008. Herbal medicine and epilepsy: Proconvulsive effects and interactions with antiepileptic drugs. Epilepsia 40: 373-380.

Santos IS, Matijasevich A, Valle NCJ 2005. Mate drinking during pregnancy and risk of preterm and small for gestational age birth. J Nutr 135: 1120-1123.

Schmidt A, Karlson-Stiber C 2008. Caffeine poisoning and lactate rise: an overlooked toxic effect? Acta Anaesthesiol Scand 52: 1012-1014. 
Seddik M, Lucidarme D, Creusy C, Filoche B 2001. Is Exolise hepatotoxic? Gastroenterol Clin Biol 25: 834-835.

Shah AH, Qureshi S, Tariq M, Aggel AM 1989. Toxicity studies on six plants used in the traditional Arab system of medicine. Phytother Res 3: 25-29.

Shulz V, Hänsel R, Tyler VE 2002. Fitoterapia Racional: Um Guia de Fitoterapia para as Ciências da Saúde. Barueri: Manole.

Siegers CP, von Hertzberg-Lottin E, Otte M, Schneider B 1993. Anthranoid laxative abuse - a risk for colorectal cancer? Gut 34: 1099-1101.

Silveira PF, Bandeira MAM, Arrais PSD 2008. Farmacovigilância e reações adversas às plantas medicinais e fitoterápicos: uma realidade. Rev Bras Farmacogn 18: 618-626.

Sossai P, Nasone C, Cantalamessa F 2007. Are herbs always good for you? A case of paralytic ileum using a herbal tisane. Phytother Res 21: 587-588.

Stewart MJ, Steenkamp V, Zuckerman M 1998. The toxicology of african herbal remedies. Ther Drug Monit 20: 510-516.

Stickel F, Patsenker E, Schuppan D 2005. Herbal hepatotoxicity. J Hepatol 43: 901-910.

Thiolet C, Mennecier D, Bredin C, Moulin O, Rimlinger H, Nizou C, Vergeau B, Farret O 2002. Acute cytolysis induced by Chinese tea. Gastroenterol Clin Biol 26: 939-940.

Tse KC, Yip PS, Lam MF, Choy BY, Li FK, Lui SL, Lo WL, Chan TM, Lai KM 2003. Star fruit intoxication in uraemic patients: case series and review of the literature. Inter Med J 33: 314-316.

Veiga-Junior VF 2008. Estudo do consumo de plantas medicinais na Região Centro-Norte do Estado do Rio de Janeiro: aceitação pelos profissionais de saúde e modo de uso pela população. Rev Bras Farmacogn 18: 308-313.

Vial T, Bernard G, Lewden B, Dumortier J, Descotes J 2003. Acute hepatitis due to exolise, a Camellia sinensisderived drug. Gastroenterol Clin Biol 27: 1166-1177.

Victora CG, Knauth DR, Hassen MNA 2000. Pesquisa qualitativa em saúde: uma introdução ao tema. Porto Alegre: Tomo Editoria.

Wong A, Castro EGR 2003. Aspectos toxicológicos dos fitoterápicos. Arq Bras Fitomed Cient 1: 96-102.

Woolf GM, Petrovic LM, Rojter SE, Wainwright S, Villamil FG, Katrov WN 1994. Acute hepatitis associated with the Chinese herbal product Jin Bu Huan. Ann Inter Med 121: 729-735.

World Health Organization (WHO) 1994. Qualitative Research for health programmes. Geneva: Division of Mental Health.

Yodyingyuad V, Bunyawong S 1991. Effect of stevioside on growth and reproduction. Hum Reprod 6: 158-165. 